\title{
A petunia mutant affected in intracellular accommodation and morphogenesis of arbuscular mycorrhizal fungi
}

\author{
Sekhara Reddy D. M. R. ${ }^{1}$, Martine Schorderet ${ }^{1}$, Urs Feller ${ }^{2}$ and Didier Reinhardt ${ }^{1, *}$ \\ ${ }^{1}$ Plant Biology, Department of Biology, University of Fribourg, Rte Albert Gockel 3, CH-1700 Fribourg, Switzerland, \\ ${ }^{2}$ Institute of Plant Science, Altenbergrain 21, CH-3013 Bern, Switzerland
}

\begin{abstract}
Summary
The regulation of the arbuscular mycorrhizal (AM) symbiosis is largely under the control of a genetic programme of the plant host. This programme includes a common symbiosis signalling pathway that is shared with the root nodule symbiosis. Whereas this common pathway has been investigated in detail, little is known about the mycorrhiza-specific regulatory steps upstream and downstream of the common pathway. To get further insight in the regulation of the AM symbiosis, a transposon-mutagenized population of Petunia hybrida was screened for mutants with defects in AM development. Here, we describe a petunia mutant, penetration and arbuscule morphogenesis1 (pam1), which is characterized by a strong decrease in colonization by three different AM fungi. Penetrating hyphae are frequently aborted in epidermal cells. Occasionally the fungus can progress to the cortex, but fails to develop arbuscules. The resulting hyphal colonization of the cortex in mutant plants does not support symbiotic acquisition of phosphate and copper by the plant. Expression analysis of three petunia orthologues of the common SYM genes LjPOLLUX, LjSYMRK and MtDMI3 indicates that pam1 is not mutated in these genes. We conclude that the PAM1 gene may play a specific role in intracellular accommodation and morphogenesis of the fungal endosymbiont.
\end{abstract}

Keywords: arbuscular mycorrhiza, arbuscular, glomus, mycorrhiza, petunia, mutant, phosphate.

Introduction

In the arbuscular mycorrhizal (AM) symbiosis between plants and soil fungi of the order Glomeromycota, the fungus penetrates either through or between cells of the root epidermis. Subsequently, the root cortex is initially colonized by intercellular hyphae, and then by the intracellular arbuscules, which give the symbiosis its name (Smith and Read, 1997). Arbuscules are finely branched hyphal structures that can fill most of the lumen of cortex cells (BonfanteFasolo, 1984), and thus provide an increased surface area for nutrient transfer between the plant and the fungus (Smith and Read, 1997).

Numerous physiological and genetic studies have provided evidence that symbiotic development is controlled by a genetic programme of the plant (Gianinazzi-Pearson, 1996; Peterson and Guinel, 2000; Marsh and Schultze, 2001; Parniske, 2004; Harrison, 2005; Stacey et al., 2006). In recent years, significant progress has been made in the elucidation of the genetic factors that regulate the AM interaction as well as the root nodule symbiosis (RNS). Genes required for both symbioses are referred to as common SYM genes. They constitute the common SYM pathway, which is required for signal transduction of the nod factor in RNS, and of a postulated myc factor in the AM interaction, respectively (Parniske, 2004; Oldroyd and Downie, 2006; Reinhardt, 2007).

In general, mutants in the common SYM genes are characterized by the early abortion of AM fungal infections either at the root surface ( $\mathrm{Pen}^{-}$phenotype) or within the root epidermal or hypodermal cells (Coi- phenotype) (GianinazziPearson, 1996; Peterson and Guinel, 2000; Marsh and Schultze, 2001; Parniske, 2004; Harrison, 2005). Several common SYM genes have been identified (Endre et al., 2002; Stracke et al., 2002; Ané et al., 2004; Lévy et al., 2004; Mitra et al., 2004; Imaizumi-Anraku et al., 2005; Kanamori et al., 2006). They encode putative signalling intermediates of the common SYM pathway, such as a receptor kinase, a putative ion channel localized to the plastids, a calcium- and calmodulin-dependent protein kinase (CCaMK) and a putative 
nucleoporin (NUP133). Homologues of common SYM genes have been found in monocot and dicot species (Ané et al., 2004; Paszkowski et al., 2006; Zhu et al., 2006), indicating that the SYM pathway may be evolutionarily conserved among all plants that are competent of AM formation.

Direct genetic screens for AM-defective mutants are necessary to identify AM-specific components, in particular the signalling components upstream and downstream of the common SYM pathway, and the structural genes required to adapt root cells to their specific function in symbiont accommodation and nutrient exchange. Such mutant screens are expected to reveal new phenotypes that differ from the described $\mathrm{Pen}^{-}$, and $\mathrm{Coi}^{-}$phenotypes. To date, only a few direct screens have been reported, namely in tomato (Barker et al., 1998; David-Schwartz et al., 2001, 2003) and maize (Paszkowski et al., 2006). They yielded an interesting new class of mutants, premycorrhizal infection (pmi) in tomato and no penetration (nope) in maize, that are affected before the first physical contact between the symbiotic partners, indicating that the earliest events of symbiotic communication upstream of the common SYM pathway may be defective.

In the AM symbiosis the plant provides essential carbohydrates to the obligate symbiotic fungus in exchange for mineral nutrients, in particular phosphate. Phosphate is thought to be taken up from the soil by extraradical hyphae, and to be transported to the roots as polyphosphate, which is depolymerized in the intraradical hyphae and then delivered to the plant (Saito, 2000; Ezawa et al., 2002). Phosphate transfer from the fungus to the plant occurs in arbusculecontaining cells over the symbiotic interface consisting of the fungal arbuscular membrane, the periarbuscular membrane of the plant and the apoplastic space in between (Karandashov and Bucher, 2005). Hallmarks of the nutrient transfer mechanism are a proton gradient generated over the membranes of the symbiotic interface by $\mathrm{H}^{+}$-ATPases (Ferrol et al., 2002), resulting in the acidification of the symbiotic interface, and the induction of phosphate transporters in the periarbuscular membrane (Harrison et al., 2002). Furthermore, fungal phosphatases are thought to be involved in the supply of phosphate to the plant (Saito, 2000; Ezawa et al., 2002; Ohtomo and Saito, 2005). Phosphatase activity and acidification of the symbiotic interface can be visualized in situ with histochemical staining techniques (Tisserant et al., 1993; Guttenberger, 2000; van Aarle et al., 2005).

Here, we describe the isolation and characterization of a Petunia mutant, penetration and arbuscule morphogenesis1 (pam 1), with specific defects in intracellular accommodation and morphogenesis of the fungal endosymbiont. The mutation results in a dramatic reduction in total root colonization and in defective arbuscule development. Metabolic staining in the root and measurements of nutrient levels in the shoot suggest that the interaction in the mutant does not contribute to the phosphate nutrition of the plant. Hence, pam 1 may be affected in the execution of a genetic programme required for proper fungal morphogenesis and for the establishment of the symbiotic machinery.

\section{Results}

\section{Isolation of the pam 1 mutant}

In order to isolate mutants affected in the development of AM, the Petunia hybrida line W138, which carries more than 100 copies of the non-autonomous transposable element dTph1 (Gerats et al., 1990), was screened. Plants were inoculated with Glomus mosseae, and the root system was harvested after 2 months and stained with a simple one-step staining protocol that reveals the extraradical mycelium. Using this method, eight individuals each of 200 families were screened for a general reduction of extraradical fungal material as a measure of a defective symbiotic interaction. Putative candidates were re-screened with the complete lactoglycerol staining procedure to reveal intraradical fungal structures. One family that contained a plant with very few extraradical hyphae was re-tested with 25 additional individuals. In this population, four mutant plants that exhibited very little mycorrhizal colonization were identified. All plants were selfed and the progeny were tested for their symbiotic phenotype. The progeny of the mutant plants consisted of mutants only, whereas five of the 21 plants with a wildtype phenotype produced only wild-type progeny. The remaining 16 families produced a segregating population with a total of 368 wild-type and 70 mutant plants. For further segregation analysis and detailed phenotypic characterization, the mutant was crossed with the stabilizer line W5 that carries an inactive allele of the master transposon ACTIVATOR1 (ACT1) (Stuurman and Kuhlemeier, 2005). The resulting F1 plants were selfed, and the segregation of the mutant phenotype was determined in the F2 generation. Among 117 F2 plants, 30 showed a mutant phenotype with severely reduced root colonization, consistent with a $3: 1$ ratio $\left(\chi^{2}=0.0256, P>0.87\right)$. Thirteen mutant plants had purely white flowers, indicating that they were homozygous for the inactive act 1 allele from W5, and that therefore the entire transposon family was stabilized. Two white-flowered mutant plants were selected for further studies. PCR analysis showed that they contained the original transposon inserted into the $A N 1$ gene (data not shown), indicating that the white-flower phenotype was indeed the result of the general inactivation of the transposon family, rather than the result of a stable footprint allele at the AN1 locus resulting from transposon excision. Both stabilized mutants produced $100 \%$ white-flowered mutant progeny. These stabilized 
mutant lines are now referred to as pam1S1 and pam1S2, respectively. The segregation among the progeny of stabilized heterozygous F2 plants was then analyzed. Eleven F3 families with 35-40 plants each exhibited segregation ratios that were consistent with a 3:1 ratio, and produced a total number of 100 mutants and 306 wild-type plants (Table S1). Taken together, these results suggest that the defect in pam 1 is caused by a single recessive mutation.

\section{AM development in wild-type P. hybrida inoculated with Glomus intraradices}

Before describing the mutant phenotype in detail, the sequence of events during AM development in wild-type petunia will be considered. For this purpose the plants were inoculated with $G$. intraradices, which was used for most of the subsequent experiments in this study. After 2 weeks the fungus had started to colonize the root surface and to form appressoria (Figure 1a). G. intraradices infected the roots either between epidermal cells or by directly penetrating epidermal cells. In the first infected cell the fungus formed a characteristic hyphal coil (Figure 1b), after which it progressed intercellularly towards the cortex where it formed the first arbuscules. From the infection site, longitudinal hyphae grew along the cortical cell files (Figure 1c). They produced lateral branches that invaded cortical cells and gave rise to secondary arbuscules (Figure 1c). After 2 months the fungus had colonized $60-90 \%$ of the entire root system with dense colonies, and had formed vesicles (not shown).

\section{Mutant phenotype of pam1}

In pam 1 mutants the fungus formed complex appressoria with many septa, indicating that it could not readily penetrate the epidermis (Figure 1d). Hyphae that penetrated an epidermal cell developed into an aggregate of distorted, sometimes bloated, hyphae, instead of a hyphal coil as in the wild type (Figure 1e, compare with Figure 1b). In many cases the hyphal clump did not develop any further, but, occasionally, the fungus was able to proceed from the epidermal cell inwards, and to colonize the cortex with longitudinal intercellular hyphae (Figure $1 \mathrm{f}, \mathrm{g}$ ). These hyphae produced lateral branches that grew through the spaces between cells (Figure 1h), and that frequently formed anastomoses (Figure 1i). Eventually, the intercellular hyphae produced many small lateral appendages (Figure 1j). This pattern of hyphal colonization sometimes comprised large sectors of the root system, with individual colonies extending over centimetres. In rare cases, extensive hyphal colonization culminated in root sectors that contained arbuscules which resembled wild-type arbuscules (data not shown).

Microscopy on Trypan Blue stained root samples indicated that hyphal colonization proceeded mostly in the extra-
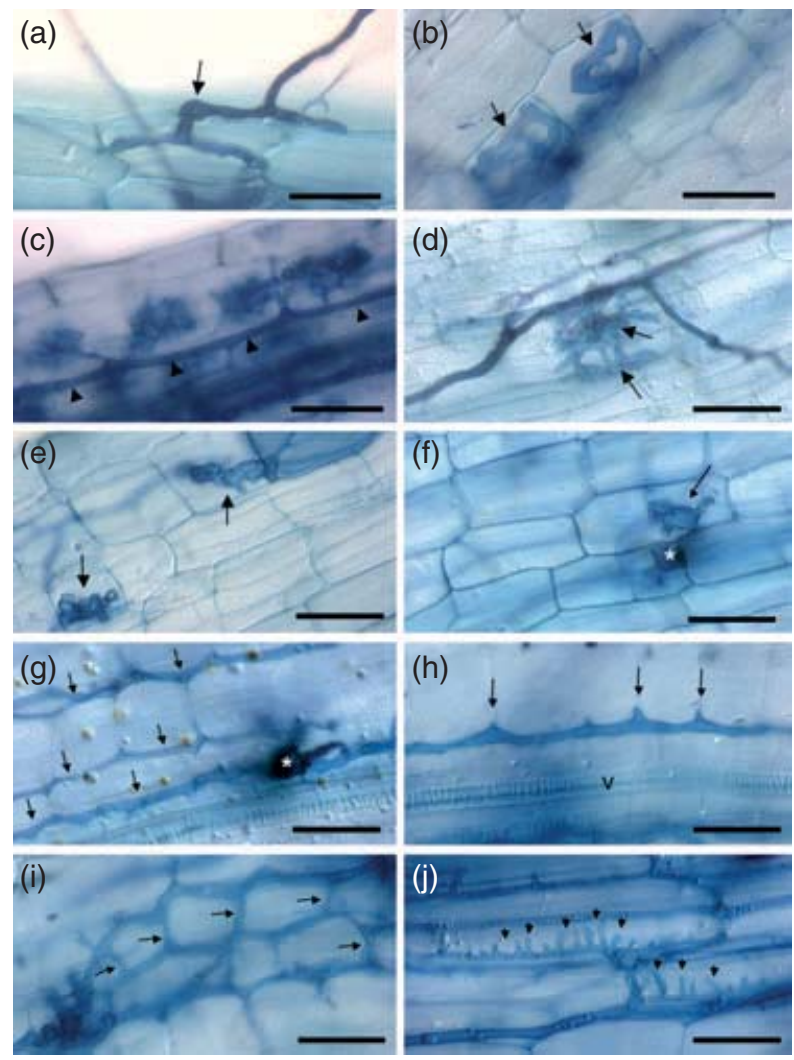

Figure 1. Development of the interaction of wild-type Petunia hybrida W138 (a-c) and the mutant pam 1 (d-j) with Glomus intraradices.

(a) An incoming extraradical hypha formed an appressorium (arrow), and subsequently penetrated an epidermal cell.

(b) After penetration, the hyphae formed one or two intracellular coils (arrows) in the epidermal cells adjacent to the penetration point.

(c) Intercellular hyphae (arrowheads) run along the root and form lateral arbuscules in cortex cells.

(d) An extraradical hypha attached itself to the root surface and formed a complex appressorium with numerous septa (arrows).

(e) Penetration of epidermal cells frequently results in clumped hyphae instead of coils.

(f and g) Same location imaged at different focal plains (*appressorium); at some entry points $(\mathrm{g})$, the fungus could penetrate further into the cortex and produce intercellular longitudinal hyphae ( $g$ and $h$, arrows).

(h) Intercellular hyphae produced lateral branches at the boundaries between cells (arrows); v, vascular strand in the central stele.

(i) Intercellular hyphae formed numerous anastomoses (arrows).

(j) Intercellular hyphae produced many lateral fine branches (arrowheads).

Scale bars $=50 \mu \mathrm{m}$.

cellular space. The exact localization of hyphal colonization, especially of the small lateral hyphae, was determined in semi-thin sections of resin-embedded material. In wild-type roots colonized cortex cells contained thick trunk hyphae and numerous fine hyphae (Figure 2a), characteristic of arbuscules (Bonfante-Fasolo, 1984). The nucleus of colonized cells was embedded in the hyphal material in a central position (Figure 2a, compare with nucleus in Figure 2b). In pam 1 mutants the intercellular spaces were occupied by thick, longitudinal hyphae, which penetrated laterally 

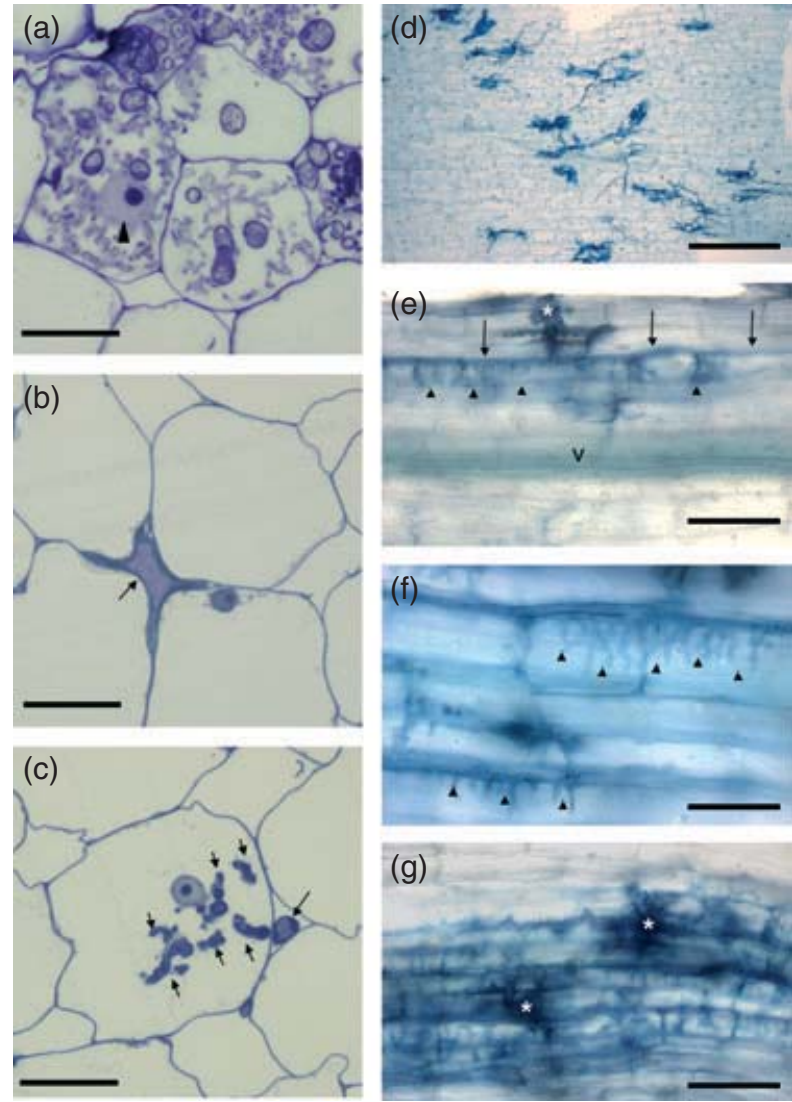

Figure 2. Inter- and intracellular colonization of the root cortex in wild-type (a) and pam 1 mutants ( $\mathrm{b}$ and $\mathrm{c}$ ), and phenotype of the stabilized line pam1S1. Plants were inoculated with Glomus intraradices, except for (d), which was inoculated with Gigaspora margarita.

(a) In wild-type cortex cells, the fungus forms thick trunk hyphae and finely branched arbuscule branches, with the host nucleus in a central position.

(b) Intercellular hypha (arrow) with branches that penetrate between cortical cells. Nuclei of the host are positioned at the periphery of the cells (arrowhead).

(c) Intercellular hypha (arrow) and intracellular colonization attempts (small arrows) in a cortex cell of pam1. The entering hyphae exhibit limited branching activity (small arrows). The host nucleus resides at a central position (arrowhead).

(d) Inoculation with G. margarita results in many aborted entry points.

(e) Despite an abnormal intracellular colonization event at the epidermis $\left({ }^{*}\right)$, $G$. intraradices was able to proceed to the cortex where it formed intercellular longitudinal hyphae (arrows) and lateral protrusions (arrowheads).

(f) Small lateral protrusions (arrowheads) of longitudinal hyphae in the cortex in a cortical cell.

(g) Large hyphal colony established from two entry points $\left({ }^{*}\right) ; \mathrm{v}$, vasculature. Scale bars: $25 \mu \mathrm{m}$ in (a)-(c), $400 \mu \mathrm{m}$ in (d), $100 \mu \mathrm{m}$ in (e) and (g), and $50 \mu \mathrm{m}$ in (f).

between cells (Figure 2b). Lateral protrusions of the intercellular hyphae extended into the cell lumen (Figure 2c, arrows), but instead of the thick and fine hyphae in wild-type arbuscules, the intracellular hyphae were of intermediate width. The plant nucleus was positioned centrally in proximity to the fungal hyphae (Figure 2c). Interestingly, the fungus produced up to 10 intracellular lateral hyphae (Figure 1j) compared with the single arbuscule that is normally formed per cell. Taken together, these observations indicate that the lateral intracellular hyphae formed in pam 1 mutants represent repeated attempts of the fungus to establish arbuscules in cortical cells. All the qualitative aspects of the pam 1 phenotype were also observed in the stabilized lines pam1S1 and pam1S2, with frequent fungal abortion at the root epidermis (Figure $2 \mathrm{~d}$ ), and with residual hyphal colonization, including lateral protrusions (Figure 2e-g). Importantly, aborted entries, as in the case of both $G$. mosseae and $G$. intraradices, were also observed when the mutant plants were inoculated with Gigaspora margarita (Figure 2d).

\section{Dynamics of the AM interaction in pam1, pam1S1 and pam1S2}

To find out how the pam 1 phenotype develops over time, a time course experiment was performed over 8 weeks (Table 1). For practical reasons, quantification of root colonization was carried out with a new method that combines the advantages of the 'root-segment method' (Trouvelot et al., 1986) with the 'grid-line intersection method' (McGonigle et al., 1990) (see Experimental procedures). In wildtype plants, total intraradical root colonization increased from 12.5 (at 3 weeks) to almost $80 \%$ (at 5 and 8 weeks). Mutants were first colonized to various degrees, from 2.5 to $32 \%$, with large variations, especially in the non-stabilized mutant (pam 1). Remarkably, all aspects of colonization decreased between 3 and 8 weeks in all three mutant lines. The number of roots with extraradical hyphae also decreased, indicating that the vitality of the fungus tapered out after prolonged co-cultivation, and that the rare colonized root sectors observed after 3 and 5 weeks became 'diluted out' by the new roots formed during the experiment. General colonization levels and the number of arbuscules were higher in the non-stable pam1 than in the stabilized lines pam1S1 and pam1S2, which were virtually devoid of arbuscules. Hence, despite the occasional success in the colonization of mutant roots, the survival of the fungus was transient, and spore production was extremely rare, suggesting that the fungus is not able to propagate in the mutants.

Besides the quantitative differences between the original mutant line (pam 1), and the stabilized line pam1S1, the lines were very similar in the qualitative aspects of their phenotype (compare Figures $1 \mathrm{~d}-\mathrm{j}$ and $2 \mathrm{~d}-\mathrm{g}$ ). All subsequent experiments, except for the nutrient measurements that were carried out only with the stabilized line pam1S1, were carried out with both lines, with comparable results. Therefore, and to avoid the impression that different alleles were used, the mutant is generally referred to in the text as pam1, and the figure legends state which line was used for the particular experiment shown. 
Table 1 The dynamics of fungal colonization in pam1

\begin{tabular}{|c|c|c|c|c|c|c|c|}
\hline 3 weeks & Extrarad. & Intrarad. & Arbuscules & Appress. & Aborted & Vesicles & Spores \\
\hline wt & $32 \pm 8.5$ & $12.5 \pm 2.1$ & $8 \pm 4.2$ & $0.5 \pm 0.7$ & 0 & $4 \pm 2.8$ & $0.5 \pm 0.7$ \\
\hline pam 1 & $31.5 \pm 3.5$ & $15 \pm 4.2$ & $3 \pm 4.2$ & $7 \pm 8.5$ & $3 \pm 4.2$ & $3.5 \pm 4.9$ & 0 \\
\hline pam1S1 & $15 \pm 5.6$ & $9 \pm 2.8$ & 0 & $8.5 \pm 2.1$ & $7 \pm 2.8$ & 0 & 0 \\
\hline pam1S2 & $9.5 \pm 0.7$ & $2.5 \pm 2.1$ & 0 & $2.5 \pm 2.1$ & $2.5 \pm 2.1$ & 0 & 0 \\
\hline 5 weeks & Extrarad. & Intrarad. & Arbuscules & Appress. & Aborted & Vesicles & Spores \\
\hline wt & $74 \pm 1.4$ & $77.5 \pm 3.5$ & $71.5 \pm 0.7$ & $28.5 \pm 2.1$ & 0 & $33.5 \pm 12.0$ & 0 \\
\hline pam 1 & $40.5 \pm 48.8$ & $31.5 \pm 44.6$ & $24 \pm 33.9$ & $10 \pm 12.7$ & $2 \pm 1.41$ & $19.5 \pm 27.6$ & 0 \\
\hline pam1S1 & $10 \pm 7.1$ & $3 \pm 2.8$ & 0 & $4 \pm 2.8$ & $4 \pm 2.8$ & 0 & 0 \\
\hline pam 1S2 & $6.5 \pm 6.4$ & $3.5 \pm 4.9$ & 0 & $2 \pm 1.4$ & $2 \pm 1.4$ & $0.5 \pm 0.7$ & 0 \\
\hline 8 weeks & Extrarad. & Intrarad. & Arbuscules & Appress. & Aborted & Vesicles & Spores \\
\hline wt & $74 \pm 11.3$ & $77.5 \pm 9.2$ & $66.5 \pm 13.4$ & $21.5 \pm 0.7$ & 0 & $29 \pm 4.2$ & $1 \pm 1.4$ \\
\hline pam 1 & $6.5 \pm 6.4$ & $5 \pm 5.6$ & 0 & $3.5 \pm 3.5$ & $3 \pm 2.8$ & $1 \pm 1.4$ & 0 \\
\hline pam1S1 & $3 \pm 0$ & 0 & 0 & $2 \pm 0$ & $2 \pm 0$ & 0 & 0 \\
\hline pam $1 S 2$ & $1.5 \pm 0.7$ & $2 \pm 0$ & 0 & 0 & 0 & 0 & 0 \\
\hline
\end{tabular}

The percentage colonization is given for three successive time points in wild type (wt), pam1, and two stabilized mutant lines (pam1S1 and pam1S2). Total extraradical (Extrarad.) and intraradical (Intrarad.) colonization, as well as the percentage of the root system with arbuscules, appressoria (Appress.), aborted entries, vesicles and spores are given.

\section{Resistance of pam 1 to nurse plant inoculation}

The nature of the inoculum can influence the outcome and the dynamics of the AM interaction in mutants. For example, the pmi mutants of tomato are resistant to fungal spores or whole-soil inoculum, including colonized roots and spores, but not to inoculation from hyphae emanating from colonized wild-type plants (nurse plants) (David-Schwartz et al., 2001, 2003). Thus, the influence of nurse plant inoculation was tested on the qualitative and quantitative aspects of the resistance of pam 1 to penetration and arbuscule development (Table 2). After 3 weeks of co-cultivation with soil inoculum, wild-type plants had extraradical hyphae on $25.7 \%$ of the root system, and $15.3 \%$ showed intraradical colonization with $12.3 \%$ arbuscular colonization $(80 \%$ relative arbuscular colonization). In pam1 plants all aspects of colonization were very low, and arbuscules were not observed. Wild-type plants exposed to nurse plants exhibited higher levels of colonization: $66 \%$ of the root system was covered with extraradical hyphae, and $72 \%$ exhibited intraradical colonization with $44.3 \%$ arbuscular colonization (61\% relative arbuscular colonization). Like wild-type plants, pam 1 plants inoculated by nurse plants had much higher colonization levels than either of the genotypes colonized from soil inoculum. The characteristic aspects, however, of the mutant phenotype were retained: intraradical colonization $(42 \%)$ remained completely devoid of arbuscules, and, frequently, the intercellular hyphae formed lateral protrusions. Both genotypes exhibited higher numbers of appressoria in the case of nurse plant inoculum compared with soil inoculum. The increased incidence of appressorium formation and intraradical root colonization indicates that infections from nurse plants impose a stronger infection pressure on wild-type and mutant plants. However, although this allowed the fungus to overcome the resistance at the epidermal barrier more frequently, and to proliferate in the cortex, it did not relieve the defect in arbuscule formation in the cortex.

\section{Specificity of the mutant phenotype}

The fact that pam 1 is resistant to three different AM fungi raised the question whether other biotic interactions may be affected as well. The response of pam 1 to three root pathogens, Phytophthora parasitica, Rhizoctonia solani and Fusarium oxysporum, was tested. Wild-type plants of the parental line W138 exhibited complete resistance to F. oxysporum, intermediate susceptibility to P. parasitica and strong susceptibility to R. solani. Young mutant and wildtype plants were inoculated with agar plugs, and the survival

Table 2 Influence of inoculum identity on the dynamics of colonization in pam1S1. Percent colonization in wild-type and pam 1S1 plants three weeks after inoculation with G. intraradices from whole-soil inoculum (soil) or from colonized wild-type plants (nurse) in separated compartments

\begin{tabular}{lllccrr}
\hline & Extrarad. & Intrarad. & Arbuscules & Appress. & aborted & Vesicles \\
\hline wt soil & $25.7 \pm 6.11$ & $15.3 \pm 2.08$ & $12.3 \pm 2.08$ & $4.67 \pm 1.15$ & $0 \pm 0$ & $8.33 \pm 2.31$ \\
Pam1S1 soil & $5.33 \pm 1.53$ & $0.67 \pm 1.15$ & $0 \pm 0$ & $0.33 \pm 0.58$ & $1.33 \pm 0.58$ & $0 \pm 0$ \\
wt nurse & $66.3 \pm 8.02$ & $72.3 \pm 8.02$ & $44.3 \pm 2.52$ & $24.3 \pm 3.51$ & $0 \pm 0$ & $5.00 \pm 1.00$ \\
Pam1S1 nurse & $48.3 \pm 4.73$ & $42.0 \pm 10.6$ & $0 \pm 0$ & $23.0 \pm 7.21$ & $7.67 \pm 2.52$ & $1.33 \pm 0.58$ \\
\hline
\end{tabular}


rate was assessed after 2 weeks. The survival rate was comparable between wild-type and pam 1, indicating that resistance and susceptibility towards the pathogens tested were not affected by the mutation in pam 1 (data not shown).

\section{Metabolic stains indicate a reduced symbiotic function in pam1}

Pam1 mutants exhibit severely reduced AM colonization levels, and the suppression of arbuscule development suggests that the supply of mineral nutrients to the plant may be defective in mutant plants. To test the vitality of the fungus and the symbiotic performance of the fungal structures in the mutant, staining procedures for metabolic functions that are thought to be relevant for the symbiosis were employed. Accumulation of the acidotropic dye Neutral Red (NR) in the periarbuscular space indicates the acidification of this compartment (Guttenberger, 2000). This observation is consistent with the idea that a proton-motive force is involved in nutrient transfer over the symbiotic interface (Ferrol et al., 2002; Karandashov and Bucher, 2005).

In petunia wild-type and pam1 roots, NR produced a general faint background staining in the root cortex, and stronger staining in the vascular stele (Figure 3a). Intercellular hyphae and the trunk hyphae of arbuscules were not stained, in contrast to the arbuscules that were frequently strongly stained (Figure 3b). In mutants, as well as in wildtype plants, intercellular longitudinal hyphae were not stained by NR. However, the small lateral protrusions that reach into cortical cells showed a weak staining (Figure 3c, compare with $3 \mathrm{~d}$ ), suggesting that the mechanism that leads to the acidification of the interface between fungus and plant may be partially active in pam 1 mutants.

Figure 3. Metabolic staining of wild-type (a, b, e, h and i) and pam 1 roots (c, d, $f, g$ and $j$ ) inoculated with Glomus intraradices.

(a-c) Neutral Red staining, (d) Trypan Blue staining, (e-g) Alkaline phosphatase activity (ALP) and ( $\mathrm{h}-\mathrm{j}$ ) Succinate dehydrogenase (SDH) activity.

(a) Weak background staining was observed in the entire root, and particularly in the vasculature (v).

(b) Arbuscules at various stages of development are stained with increasing intensity (arrows) until maturity $\left({ }^{*}\right)$.

(c) In pam 1 roots, the intercellular longitudinal hyphae (arrow) were not stained, whereas the small intracellular protrusion (arrowheads) exhibited weak staining.

(d) Trypan Blue staining of the same root as in (c), with arrow and arrowheads placed at identical positions to those in (c). Note staining of the longitudinal hypha (arrow).

(e) High ALP activity was found in the arbuscules (white asterisks), but some arbuscules were only weakly stained (black asterisks).

(f) In pam 1 penetrating hyphae (arrow) exhibited weak ALP staining, whereas intercellular hyphae (arrowheads) were not stained.

(g) Lateral protrusions (arrowheads) have no ALP staining.

(h) Fungal hyphae (arrows) generally exhibit SDH staining.

(i) Arbuscules $\left({ }^{*}\right)$ consistently exhibit strong or intermediate SDH staining. (j) Intercellular longitudinal hyphae and lateral protrusions exhibit strong SDH staining (arrowheads).

Scale bars: $100 \mu \mathrm{m}(\mathrm{a}) ; 50 \mu \mathrm{m}(\mathrm{b}-\mathrm{j})$.
The general reduction in arbuscule development and interface acidification could be associated with a decrease in fungal vitality. To test this, the activity of fungal alkaline phosphatase (ALP) and succinate dehydrogenase (SDH), as markers for metabolic activity, were assessed (Tisserant et al., 1993; van Aarle et al., 2005). In wild-type plants ALP activity was detected in intraradical hyphae (not shown) and in the arbuscules, which showed the strongest signal (Figure 3e), although not all of them were stained at the same level. In mutant roots the first intracellular hyphae in the epidermis showed a weak ALP staining (Figure 3f), but neither the longitudinal nor the small lateral protrusions were stained in pam1 (Figure $3 \mathrm{~g}$ ).
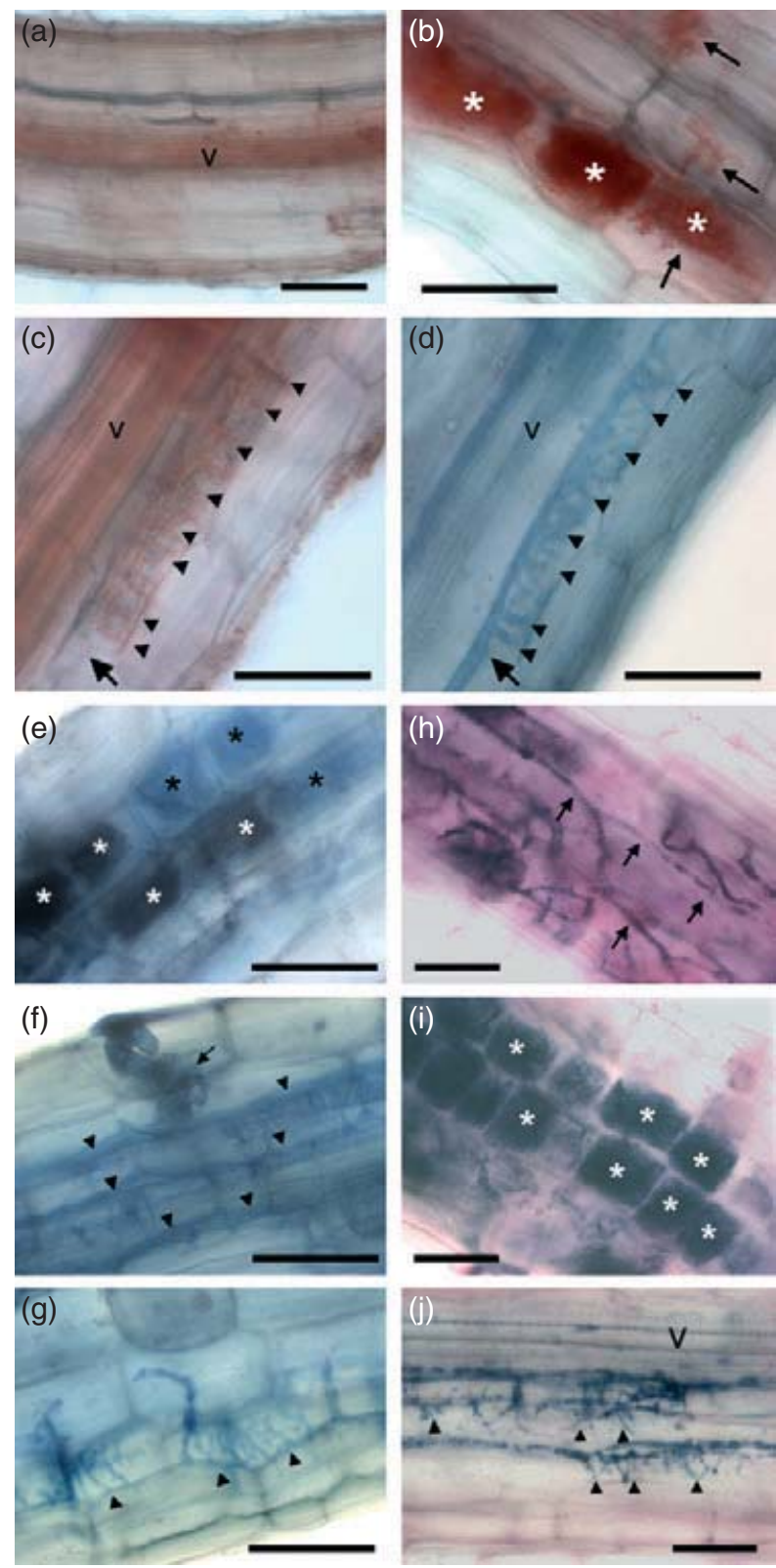
SDH-activity staining was then carried out to see whether the low levels of ALP staining in mutants reflected a general reduction in the metabolic activity of the fungus. In wild-type roots SDH activity was detected in various fungal structures, including intercellular hyphae (Figure $3 \mathrm{~h}$ ) and arbuscules (Figure 3i). In mutant roots $\mathrm{SDH}$ staining was observed in the longitudinal hyphae, as well as in the fine lateral branches (Figure 3j). Thus, the low levels of ALP activity in the fungal hyphae found in pam 1 mutant plants do not result from a general reduction of metabolic activity of the fungus.

\section{Nutrient levels in mutant and wild-type plants}

Taken together, our results suggest that nutrient exchange in colonized pam 1 plants may be defective. To test this possibility, the effect of AM colonization on the levels of mineral nutrients in the shoot of mutant and wild-type plants was analyzed. After 8 weeks of co-culture with G. intraradices, $\mathrm{P}$ and $\mathrm{Cu}$ levels were higher by approximately three- and twofold, respectively, in mycorrhizal wild-type plants, compared with non-mycorrhizal controls (Figure $4 a, b$ ), whereas the levels of $\mathrm{Zn}, \mathrm{Mg}, \mathrm{Ca}$ and $\mathrm{K}$ were
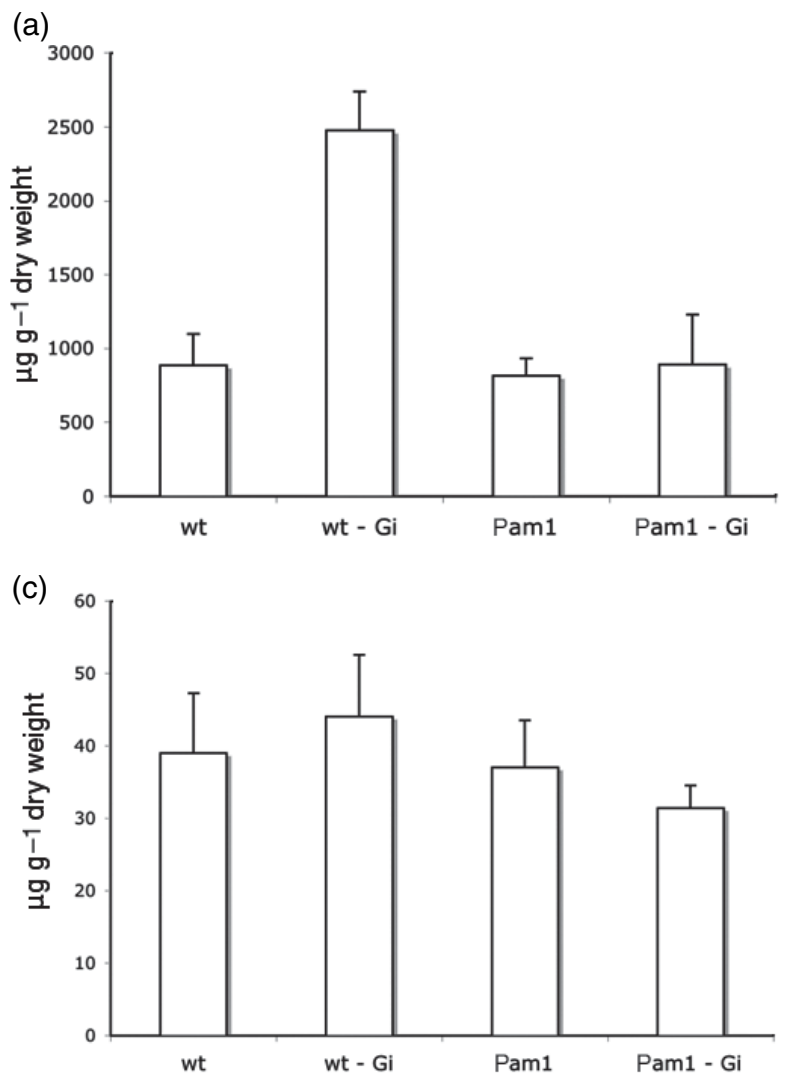

not significantly altered in inoculated roots, compared with controls (Figure 4c, and data not shown). In contrast to wild-type plants, the levels of $\mathrm{P}$ and $\mathrm{Cu}$ in mutant shoots were not affected by the presence of $G$. intraradices, and were not significantly different from wild-type control plants (Figure $4 a, b$ ). In order to find out whether small differences in the $P$ levels of individual mutant plants correlated with hyphal colonization in the root cortex, the level of $P$ in the mutant shoots was plotted against the level of fungal colonization (Figure 4d). No correlation, however, between shoot $\mathrm{P}$ levels and root colonization could be detected (Pearson correlation coefficient $=-0.09$ ). Taken together, these data suggest that the hyphal colonization in pam 1 does not contribute to $\mathrm{P}$ and $\mathrm{Cu}$ nutrition of the shoot.

\section{Expression in pam1 of the petunia orthologues of common SYM genes}

Some aspects of the pam 1 mutant phenotype are reminiscent of phenotypes of mutants in common SYM genes (Demchenko et al., 2004), indicating that pam1 might carry a mutation in a petunia SYM gene homologue. To test this (b)

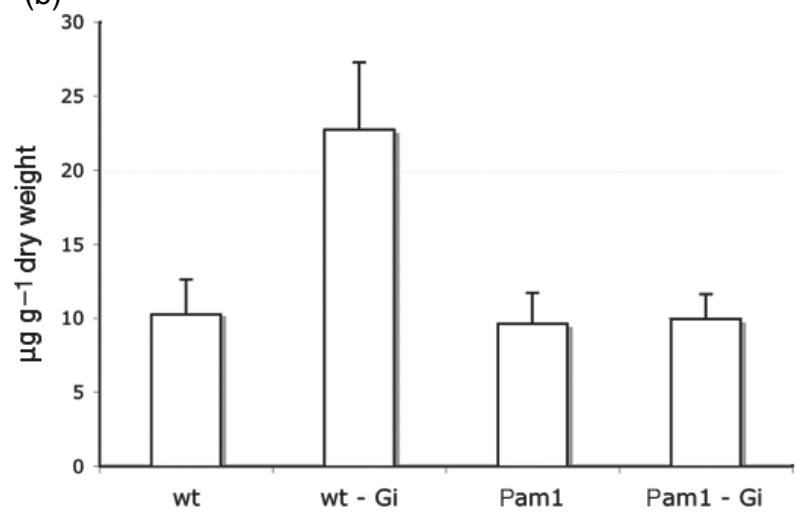

(d)

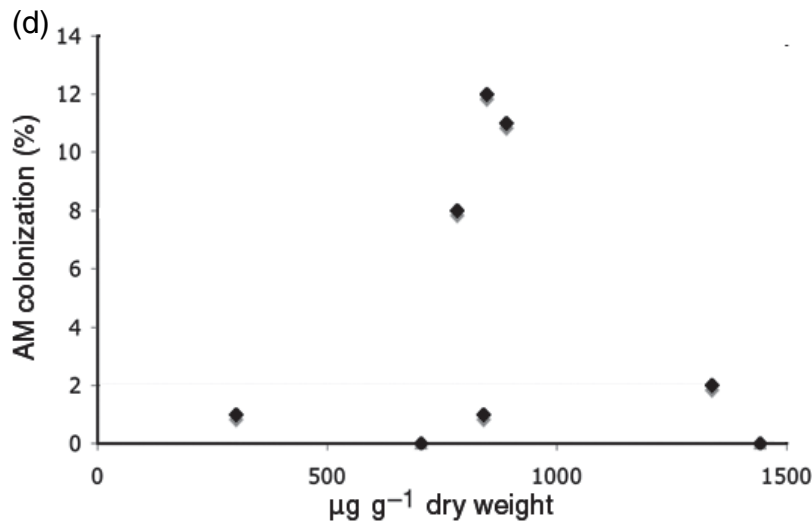

Figure 4. Mineral nutrient content in wild-type and pam 1S1 mutant shoots 8 weeks after inoculation with soil inoculum of Glomus intraradices. (a) P content, (b) Cu content and (c) Zn content.

(d) Correlation between $\mathrm{P}$ content in the shoot and hyphal colonization in the root of pam1S1. 
possibility, we cloned petunia cDNA sequences with high homology to the SYMBIOSIS RECEPTOR KINASE (SYMRK) and POLLUX genes of Lotus japonicus (Stracke et al., 2002; Imaizumi-Anraku et al., 2005) and to the Medicago truncatula common SYM gene DOES NOT MAKE INFECTIONS3 (DMI3) (Lévy et al., 2004; Mitra et al., 2004) (see Experimental procedures). The petunia genes PhSYMRK, PhPOL$L U X$ and $P h D M I 3$ (accession numbers EF613119, EF613118, and EF592572, respectively), will be described in detail elsewhere. The receptor kinase PhSYMRK, and PhDMI3, which encodes a CCaMKs, were more related to the respective legume SYM gene homologues than to any other receptor kinase or CCaMK from any species (including Solanaceae), indicating that they represent the petunia orthologues of the legume SYM genes. Attempts to isolate petunia homologues of NUP133 (Kanamori et al., 2006) and CASTOR (Imaizumi-Anraku et al., 2005) were not successful (data not shown).

Transposon activity can affect gene expression in two ways: (i) insertions in the promoter region can interfere directly with transcription, and (ii) insertion in the coding region can lead to frame shifts and premature termination codons. Such defective transcripts are subject to degradation by a conserved mechanism referred to as nonsensemediated mRNA decay (NMD) (Weischenfeldt et al., 2005), which operates in many eukaryotes including plants (ArcigaReyes et al., 2006). Based on the assumption that NMD would purge the transcripts of genes mutated by transposon insertion, a maize mutant has recently been checked for potential defects in the expression of several SYM gene homologues (Paszkowski et al., 2006).

The transcript levels of PhSYMRK, PhPOLLUX and $P h D M I 3$ were determined by real-time reverse-transcriptase polymerase chain reaction analysis with GAPDH as a reference. The transcript level of all three genes was slightly lower in pam 1 mutant roots compared with wild-type roots (Table 3), with only PhSYMRK exhibiting a significant difference (Student's $t$-test). Taken together these results suggest that the defect in pam 1 is not the result of a transposon insertion in the petunia SYM gene homologues tested here, whereas a general indirect effect of the pam 1

Table 3 Relative expression of petunia SYM genes in wild-type and pam1S1 roots. Expression levels of PhPOLLUX, PhSYMRK and $P h D M / 3$ are expressed relative to GAPDH expression. Wild-type gene expression was normalized to 100

\begin{tabular}{lll}
\hline & wt & pam1S1 \\
\hline PhPOLLUX & $100 \pm 16.4$ & $75.5 \pm 8.93$ \\
PhSYMRK & $100 \pm 22.0^{*}$ & $71.8 \pm 7.84^{*}$ \\
PhDMI3 & $100 \pm 15.3$ & $86.4 \pm 11.8$ \\
\hline
\end{tabular}

*Expression of PhSYMRK is significantly lower in pam1S1 compared with controls. mutation on the expression of the SYM genes cannot be excluded. However, it seems unlikely that a small reduction in transcript levels of $15-30 \%$ could be responsible for the symbiotic defects observed in pam 1 .

\section{Discussion}

Pam 1 is defective in intracellular accommodation and fungal morphogenesis

Screening of a transposon-mutagenized petunia line for mutants affected in the development of the AM symbiosis yielded the mutant pam1. The pam 1 mutant exhibits defects in the penetration of the root epidermis and the invasion of the cortex, resulting in the early abortion of many invading hyphae, and in a strong decrease of general root colonization, just as in most legume mutants and in $r m c$ of tomato (Barker et al., 1998). In cases where the fungus was able to invade the cortex it colonized the root with a profuse intercellular hyphal network, but arbuscule formation was blocked. Instead, many short intracellular hyphae were formed as lateral protrusions of the intercellular hyphae. The fact that intracellular hyphal structures were found, both in epidermal and cortical cells, suggests that the defect of pam 1 is not in the process of cellular penetration per se, but rather in an accommodation programme that allows the fungus to form the appropriate intracellular structures: coils in the epidermis and arbuscules in cortical cells. Weak mutant alleles of common SYM genes have also been shown to display dual defects at root penetration and at arbuscule formation (Novero et al., 2002; Demchenko et al., 2004). In these cases, however, the formation of lateral protrusions of intercellular hyphae in the cortex was not reported. In this context it is interesting to note that defects in the phosphate transport machinery also lead to defects in arbuscule development, indicating that symbiotic development is subject to complex regulatory feedback mechanisms (Maeda et al., 2006; Javot et al., 2007).

In a time course experiment with soil inoculum, the hyphal colonization of pam 1 plants was low after 3 weeks, decreased until 8 weeks after inoculation, and ultimately virtually no fungal colonization was observed (Table 1). In contrast, infection of pam 1 from wild-type 'nurse plants' led to much higher levels of colonization (Table 2). Hyphae emanating from inoculated nurse plants are considered to impose a stronger infection pressure because they enjoy a continuous supply of nutrients and energy, whereas the fungal propagules from soil inoculum have a limited infectivity if they do not reach a host within a few weeks. Importantly, despite the strong 'virulence' of the nurse plant inoculations, the colonization remained hyphal with lateral protrusions, and arbuscules were not observed. This indicates that the requirement for PAM1 function in the 
penetration of the root epidermis may vary depending on the nature of the inoculum, whereas $P A M 1$ is indispensable for arbuscule development in the cortex irrespective of the nature of the inoculum.

\section{Specificity of the pam 1 mutant phenotype}

Specificity in the AM interaction is very low, i.e. most plant species that are competent of AM formation are compatible with many AM fungal isolates under experimental conditions (Smith and Read, 1997). If a general component required for symbiotic interactions was affected in pam1, general resistance to various AM fungi, or even to fungal pathogens, could result. Three AM fungi, G. intraradices (MUCL 43204), G. mosseae (BEG12) and G. margarita (BEG $34)$, were tested for their ability to colonize pam1, and three pathogens, $P$. parasitica, $R$. solani and F. oxysporum were tested to discover if the mutant was altered in its resistance to root pathogens. Although the parental line W138 was colonized by all three mycorrhizal fungi, pam 1 plants were resistant to all three AM fungi at a similar level, suggesting that the defect of pam 1 is not specific for a particular AM fungal species or isolate. In the case of inoculations with the pathogens, pam1, like W138, was strongly susceptible to $R$. solani, intermediately susceptible to $P$. parasitica and fully resistant to F. oxysporum. Thus, the mutation in PAM1 did not alter the resistance or susceptibility to these three pathogens. This observation is consistent with the observation that various plant-pathogen interactions are not affected by mutations in common SYM genes (Gianinazzi-Pearson et al., 1994; Mellersh and Parniske, 2006).

\section{Functional aspects of $A M$ development in pam 1}

The observed defects in fungal development in pam 1 raised the question of how this relates to symbiotic functions. The reduced levels of general colonization and the strong reduction of hyphal branching in cortex cells of pam 1 plants are likely to reduce the surface area available for nutrient transfer. In addition, staining with the acidotropic dye NR indicated that the interface in cortical cells was less acidified than in wild-type cells inhabited by arbuscules. Apoplast acidification, which is thought to result from $\mathrm{H}^{+}$-ATPase activity at the periarbuscular or arbuscular membrane, might serve to energize the nutrient transfer over the symbiotic interface (Guttenberger, 2000; Ferrol et al., 2002). Similarly, the ALP activity of the intraradical hyphal material in pam 1 was lower than that of arbuscules in the wild type, whereas fungal vitality (based on SDH staining) was comparable in mutant and wild-type plants. It has been hypothesized that fungal ALP activity, which is induced in the context of the host, could be indicative of an efficient phosphate supply to the plant (Tisserant et al.,
1993). Based on these assumptions, our results indicate that pam 1 mutants may lack a signal required for ALP induction, and that the residual hyphal colonization in pam 1 mutants is characterized by reduced levels of symbiotic functions.

To assess symbiotic benefits to the plant more directly, fungal inoculations were tested for their ability to improve the nutrient status in wild type and pam 1 mutants. In the wild type, $\mathrm{AM}$ colonization increased the level of $\mathrm{P}$ and $\mathrm{Cu}$ in the shoot, whereas $\mathrm{Zn}, \mathrm{Mg}, \mathrm{Ca}$ and $\mathrm{K}$ levels were not significantly affected (Figure 4). In contrast, $\mathrm{P}$ and $\mathrm{Cu}$ levels were not increased in pam 1 plants in the presence of $G$. intraradices, and the variations of $P$ content among the eight pam 1 replicate plant samples did not correlate with the level of hyphal colonization in the roots of these plants. These observations suggest that the residual hyphal colonization in pam 1 mutants does not contribute to $\mathrm{P}$ and $\mathrm{Cu}$ nutrition of the shoot.

\section{Are SYM genes defective in pam1?}

Abortion of fungal entries in epidermal cells is a common feature of pam 1 and mutants in common SYM genes (Parniske, 2004). It was therefore important to test whether the function of SYM genes might be compromised in pam 1. Partial sequences of the petunia SYM genes PhSYMRK, PhPOLLUX and PhDMI3 were cloned, and their expression quantified. The transcript levels of these three genes were reduced by $15-30 \%$ in pam 1 compared with wild-type plants, and only in the case of PhSYMRK was the difference significant (Table 3). Such a minimal difference is not sufficient to explain the mutant phenotype of pam1. Taken together, these results do not point to a transposon insertion in the three petunia SYM genes tested here. However, a moderate indirect effect of the pam 1 mutation on SYM gene expression cannot be excluded.

\section{Conclusion}

We describe the isolation and characterization of a petunia mutant, pam 1, which has a dual defect in the development of AM symbiosis. Fungal colonization of pam 1 roots is blocked at the intracellular steps in the root epidermis and the cortex, whereas intercellular hyphal growth appears not to be affected. Although the defects in root penetration and arbuscule formation are also observed in some mutants of common SYM genes, the formation of numerous lateral protrusions in the root cortex is unique to pam1. Functional analysis of the interaction in mutants suggests that the fungus does not confer a nutritional benefit to the plant. The combined evidence indicates that the mutant may be affected in a gene with a function in intracellular accommodation and morphogenetic control of the fungal endosymbiont. 


\section{Experimental procedures}

\section{Plant growth and inoculation}

Petunia W138 plants were germinated on sterilized seedling substrate (Klasmann, http://www.klasmann-deilmann.com). After 4 weeks plantlets were transferred to a sterilized mixture of $50 \%$ sand with $50 \%$ unfertilized soil, and inoculated with 1 teaspoon $(c .10 \mathrm{~g}$ ) of inoculum per plant directly to the root system. Plants for screening were grown in a glasshouse; plants for time course and for physiological experiments were grown in growth chambers with a day/night cycle of $16\left(25^{\circ} \mathrm{C}\right)$ and $8 \mathrm{~h}\left(20^{\circ} \mathrm{C}\right)$. Plants were fertilized weekly with a solution containing the following nutrients: $3 \mathrm{~mm} \mathrm{MgSO}_{4}, 0.75 \mathrm{~mm} \mathrm{KNO}_{3}, 0.87 \mathrm{~mm} \mathrm{KCl}, 0.2 \mathrm{~mm} \mathrm{KH}_{2} \mathrm{PO}_{4}$, $1.52 \mathrm{~mm} \mathrm{Ca}\left(\mathrm{NO}_{3}\right)_{2}, 0.02 \mathrm{~mm} \mathrm{NaFeEDTA}, 11 \mathrm{~mm} \mathrm{MnSO}_{4}, 1 \mathrm{~mm}$ $\mathrm{ZnSO}_{4}, 30 \mathrm{mM} \mathrm{H}_{3} \mathrm{BO}_{3}, 0.96 \mathrm{~mm} \mathrm{CuSO}, 0.03 \mathrm{~mm}\left(\mathrm{NH}_{4}\right)_{6} \mathrm{Mo}_{7} \mathrm{O}_{24}$ and $0.01 \mathrm{mM} \mathrm{Na}_{2} \mathrm{MoO}_{4}$. Nurse plant inoculations were carried out in dual compartment systems where the inoculated wild-type plants were separated from the pam 1 mutants by a $50 \mu \mathrm{m}$ nylon mesh (Wyss et al., 1991). In the case of inoculations with pathogens, the young plants were inoculated at the time of transplanting to the sand/soil mixture with four small agar plugs placed next to the roots.

\section{Fungal inoculum}

Inoculum of G. mosseae (BEG12), G. margarita (BEG34), and G. intraradices (MUCL 43204), respectively, was produced in leek pot cultures that were at least 6 -months old, and had been tested for the presence of spores. The inoculum consisted of a mixture of soil and roots. Inoculum of $P$. parasitica (strain 237), R. solani (strain CBS 101772) and F. oxysporum Schlechtend.:Fr. (Mauch-Mani and Slusarenko, 1994) was produced on plates with V8 agar ( $P$. parasitica and $R$. solani) or potato dextrose agar (F. oxysporum).

\section{Staining procedures}

Trypan Blue staining. For the primary mutant screening, roots were harvested, washed and stained for $10 \mathrm{~min}$ in $0.5 \%$ acetic acid/ $0.01 \%$ Trypan Blue at $95^{\circ} \mathrm{C}$, and washed with water. For time course experiments and re-screening, roots were cleared in $10 \% \mathrm{KOH}$ (30 min at $95^{\circ} \mathrm{C}$ ), washed twice with water, stained for 10 min with Trypan Blue staining solution at $95^{\circ} \mathrm{C}(20 \%$ glycerol, $30 \%$ lactic acid and $0.01 \%$ Trypan Blue) and rinsed twice with $30 \%$ lactic acid.

Neutral Red staining. Neutral Red staining was performed with $10 \mu \mathrm{M} \mathrm{NR}$ in $10 \mathrm{~mm} 3$-( $N$-morpholino)propanesulphonic acid (MOPS)/KOH, pH 7.3, in the dark overnight (Guttenberger, 2000). SDH activity staining was performed as previously described (Kough et al., 1987); ALP activity staining was performed according to the method described by Tisserant et al. (1993). Thereafter, the roots were washed three times and cleared with $10 \% \mathrm{KOH}$ for $15 \mathrm{~min}\left(95^{\circ} \mathrm{C}\right)$. After two washes, the roots were counterstained with Trypan Blue staining solution (see above). All staining experiments were conducted with non-stabilized and stabilized mutants, with similar results.

Microscopy. Images were acquired using a Zeiss Axiocam (http:// www.zeiss.com) mounted on a Leica DMR microscope (http:// www.leica-microsystems.com) equiped with Nomarski optics. For semi-thin sections, root segments were fixed in $0.1 \mathrm{~m} \mathrm{Na-cacodylate,}$
$\mathrm{pH} 7.4$, with $4 \%$ glutaraldehyde for $2 \mathrm{~h}$ at $20^{\circ} \mathrm{C}$. After overnight postfixation with $1 \% \mathrm{OsO}_{4}$ in $0.1 \mathrm{M} \mathrm{Na}$-cacodylate at $4^{\circ} \mathrm{C}$, samples were dehydrated through an acetone series, embedded in Spurr's standard epoxy resin and polymerized at $70^{\circ} \mathrm{C}$ for $18 \mathrm{~h}$. Semi-thin sections (1- $\mu \mathrm{m}$ thick) were prepared with a diamond knife on a Reichert-Jung microtome (Ultracut E; http://www.leica-microsystems.com).

Quantification of mycorrhizal colonization. For quantification of root colonization, a new method was used that combines the advantages of the 'root-segment method', which assesses the percentage of colonized root segments in a sample of root pieces of defined size (Trouvelot et al., 1986), and the 'grid-line intersection method', which assesses the presence of fungal structures at intersections of the roots with a grid system (McGonigle et al., 1990). The highly ramified thin roots of petunia cannot easily be cut in regular pieces of a defined length, thereby preventing the adoption of the first method. On the other hand, the infinitesimally small sample size (a line intersection) makes it difficult to quantify rare structures, especially in mutants. Thus, it was desirable to sample a defined number of small root segments at random positions in the root system. To this end a grid of yellow vertical lines, with a defined width of $0.5 \mathrm{~mm}$ and a horizontal spacing of $5 \mathrm{~mm}$, was printed on ink-jet transparency foils. The transparency was then cut in strips and taped onto glass slides with the printed side down (with the yellow lines in a vertical orientation), and the root samples were spread horizontally over the transparency. The presence of the various fungal structures was recorded by determining the intersection areas of the blue roots with the yellow grid. Hence, the $0.5-\mathrm{mm}$ segments per $5-\mathrm{mm}$ root length represent a random sample of $10 \%$ of the entire root sample. Per sample, 100 intersections were assessed. The occurrence of intraradical hyphae (Intrarad. in Table 1) is defined as all the events in which any intraradical structures were observed, including arbuscules or vesicles, because these structures are always associated with hyphae. Scores for arbuscules and vesicles represent all the instances in which the intraradical colonization featured the respective structure. Subtraction of the arbuscule values from the intraradical hyphae value yields the percentage of root segments that contained only hyphae and vesicles. Relative arbuscular colonization is the ratio of arbuscular to total intraradical colonization.

Determination of shoot nutrient levels. Plants were harvested, the roots used for the quantification of AM colonization and the shoots were dried at $120^{\circ} \mathrm{C}$. Dry leaves were weighed and dry ashed at $550^{\circ} \mathrm{C}$ overnight, and dissolved in $35 \% \mathrm{HCl}$. Further processing and measurements by atomic absorption spectrometry were carried out as described previously (Stieger and Feller, 1994).

\section{DNA isolation and cloning of Petunia SYM gene homo-} logues. Petunia genomic DNA was isolated using the protocol of Murray and Thompson (1980). Briefly, $1 \mathrm{~g}$ of leaf material was ground in liquid nitrogen and dissolved in $5 \mathrm{ml}$ grinding buffer ( $350 \mathrm{~mm}$ sorbitol, $100 \mathrm{~mm}$ Tris- $\mathrm{HCl}, \mathrm{pH} 7.5,10 \mathrm{~mm}$ EDTA, pH 8.0) and centrifuged at $14000 \times \mathrm{g}$ for $10 \mathrm{~min}$ at $4^{\circ} \mathrm{C}$. The pellet was dissolved in $5 \mathrm{ml}$ of pre-warmed lysis buffer $(200 \mathrm{~mm}$ Tris-HCl, $\mathrm{pH} 7.5,50$ mm, EDTA, pH 8.0, 2 м sodium chloride, $2 \%$ W/N cetyltrimethylammonium bromide (CTAB)) and incubated at $65^{\circ} \mathrm{C}$ with occasional shaking for $1 \mathrm{~h}$ in a water bath. After extraction with chloroform:isoamyl alcohol (24:1), the DNA was precipitated with isopropanol.

For the isolation of SYM gene homologues, degenerated primers were designed according to the conserved nucleotides and amino acid sequences of $L$. japonicus SYMRK and POLLUX, and of 
M. truncatula DMI3. PCR was performed on genomic DNA and cDNA. Resulting amplicons were gel-eluted by using the QIAquick gel elution kit (Qiagen, http://www.qiagen.com) and cloned into pGEMT-E vector (Promega, http://www.promega.com).

RNA isolation, first-strand cDNA synthesis and quantitative real-time PCR. Total RNA was isolated using the hot phenol method (Verwoerd et al., 1989). Total RNA was treated with DNAse I according to the protocol of the provider (Qiagen). Total RNA (1 $\mu \mathrm{g}$ ) was reverse transcribed (Omniscript RT kit; Qiagen). qPCR was performed with the ABsolute QPCR SYBR Green mix (ABgene) on a Rotor-Gene 2000 cycler (Corbett Research, http://www. corbettlifescience.com). The following primers were used: 5 '-CACCCAGGCCAAGAAATGAATGGAGCT- $3^{\prime}$ and $5^{\prime}$-CTCCACAAACTTCTC AGAGAAATCTGTTGCC-3' for PhSYMRK， 5'-CAAGAGCATCGGT GGTGGTGTCGTTGTTGTGCTCGC-3' and '5'-CACTCATCTCCACCACAACATGACCTCTCAACCCCTC-3' for PhPOLLUX, and 5'-CACCGTTGGTTCCTATGACCTCAAGCCTG- $3^{\prime}$ and $5^{\prime}$-CGAAGAGAGG AGAGAACTACATCTTGAAGAGAGC-3' for PhDMI3.

\section{Acknowledgements}

We thank Pierre Ricci, Thomas Boller, Brigitte Mauch-Mani and Raffaella Balestrini for providing fungal strains, Jeroen Stuurman for providing the petunia line W5, Cris Kuhlemeier for stimulating discussions, and Paola Bonfante, Jean-Pierre Métraux and Antony Buchala for critical reading of the manuscript. This research was supported by the Swiss National Science Foundation (Grant No. 3100A0-101792).

\section{References}

van Aarle, I.M., Cavagnaro, T.R., Smith, S.E., Smith, F.A. and Dickson, S. (2005) Metabolic activity of Glomus intraradices in Arum- and Paris-type arbuscular mycorrhizal colonization. New Phytol. 166, 611-618.

Ané, J.-M., Kiss, G.B., Riely, B.K. et al. (2004) Medicago truncatula DMI1 required for bacterial and fungal symbioses in legumes. Science, 303, 1364-1367.

Arciga-Reyes, L., Wootton, L., Kieffer, M. and Davies, B. (2006) UPF1 is required for nonsense-mediated mRNA decay (NMD) and RNA in Arabidopsis. Plant J. 47, 480-489.

Barker, S.J., Stummer, B., Gao, L., Dispain, I., O'Connor, P.J. and Smith, S.E. (1998) A mutant in Lycopersicon esculentum Mill. with highly reduced VA mycorrhizal colonization: isolation and preliminary characterisation. Plant J. 15, 791-797.

Bonfante-Fasolo, P. (1984) Anatomy and morphology of VA mycorrhizae. In VA Mycorrhizae, Vol. 57 (Powell, C.L. and Bagyaraj, D.J., eds). Boca Raton, FL, USA: CRC Press, pp. 5-33.
David-Schwartz, R., Badani, H., Smadar, W., Levy, A.A., Galili, G. and Kapulnik, Y. (2001) Identification of a novel genetically controlled step in mycorrhizal colonization: plant resistance to infection by fungal spores but not extra-radical hyphae. Plant $J$. 27, 561-569.

David-Schwartz, R., Gadkar, V., Wininger, S., Bendov, R., Galili, G., Levy, A.A. and Kapulnik, Y. (2003) Isolation of a premycorrhizal infection (pmi2) mutant of tomato, resistant to arbuscular mycorrhizal fungal colonization. Mol. Plant Microbe Interact. 16, 382-388.

Demchenko, K., Winzer, T., Stougaard, J., Parniske, M. and Pawlowski, K. (2004) Distinct roles of Lotus japonicus SYMRK and SYM15 in root colonization and arbuscule formation. New Phytol. 163, 381-392.

Endre, G., Kereszt, A., Kevei, Z., Mihacea, S., Kaló, P. and Kiss, G.B. (2002) A receptor kinase gene regulating symbiotic nodule development. Nature, 417, 962-966.

Ezawa, T., Smith, S.E. and Smith, F.A. (2002) P metabolism and transport in AM fungi. Plant Soil, 244, 221-230.

Ferrol, N., Gianinazzi, S. and Gianinazzi-Person, V. (2002) Arbuscular mycorrhiza induced ATPases and membrane nutrient transport mechanisms. In Mycorrhizal Technology in Agriculture: From Genes to Bioproducts (Gianinazzi, S., Schüepp, H., Barea, J.M. and Haselwandter, K., eds). Basel: Birkhäuser, pp. 113-122.

Gerats, A.G.M., Huits, H., Vrijlandt, E., Maraña, C., Souer, E. and Beld, M. (1990) Molecular characterization of a nonautonomous transposable element (dTph1) of petunia. Plant Cell, 2, 1121-1128.

Gianinazzi-Pearson, V. (1996) Plant cell responses to arbuscular mycorrhizal fungi: getting to the roots of the symbiosis. Plant Cell, 8, 1871-1883.

Gianinazzi-Pearson, V., Gollotte, A., Dumas-Gaudot, E., Franken, P. and Gianinazzi, S. (1994) Gene expression and molecular modifications associated with plant responses to infection by arbuscular mxcorrhizal fungi. In Advances in Molecular Genetics of Plant-Microbe Interactions, Vol. 3 (Daniels, M.J., Downie, J.A and Osbourn, A.E., eds). Dordrecht: Kluwer Academic Publishers, pp. 179-186.

Guttenberger, M. (2000) Arbuscules of vesicular-arbuscular mycorrhizal fungi inhabit an acidic compartment within plant roots. Planta, 211, 299-304.

Harrison, M.J. (2005) Signaling in the arbuscular mycorrhizal symbiosis. Annu. Rev. Microbiol. 59, 19-42.

Harrison, M.J., Dewbre, G.R. and Liu, J.Y. (2002) A phosphate transporter from Medicago truncatula involved in the acquisiton of phosphate released by arbuscular mycorrhizal fungi. Plant Cell, 14, 2413-2429.

Imaizumi-Anraku, H., Takeda, N., Charpentier, M. et al. (2005) Plastid proteins crucial for symbiotic fungal and bacterial entry into plant roots. Nature, 433, 527-531.

Javot, H., Penmetsa, R.V., Terzaghi, N., Cook, D.R. and Harrison, M.J. (2007) A Medicago truncatula phosphate transporter indispensable for the arbuscular mycorrhizal symbiosis. Proc. Natl Acad. Sci. USA, 104, 1720-1725.

Kanamori, N., Madsen, L.H., Radutoiu, S. et al. (2006) A nucleoporin is required for induction of $\mathrm{Ca} 2+$ spiking in legume nodule development and essential for rhizobial and fungal symbiosis. Proc. Natl Acad Sci USA, 103, 359-364.

Karandashov, V. and Bucher, M. (2005) Symbiotic phosphate transport in arbuscular mycorrhizas. Trends Plant Sci. 10, 22-29.

Kough, J.L., Gianinazzi-Pearson, V. and Gianinazzi, S. (1987) Depressed metabolic activity of vesicular-arbuscular mycorrhizal fungi after fungicide applications. New Phytol. 106, 707-715. 
Lévy, J., Bres, C., Geurts, R. et al. (2004) A putative $\mathrm{Ca}^{2+}$ and calmodulin-dependent protein kinase required for bacterial and fungal symbioses. Science, 303, 1361-1364.

Maeda, D., Ashida, K., Iguchi, K., Chechetka, S.A., Hijikata, A., Okusako, Y., Deguchi, Y., Izui, K. and Hata, S. (2006) Knockdown of an arbuscular mycorrhiza-inducible phosphate transporter gene of Lotus japonicus suppresses mutualistic symbiosis. Plant Cell Physiol. 47, 807-817.

Marsh, J.F. and Schultze, M. (2001) Analysis of arbuscular mycorrhizas using symbiosis-defective plant mutants. New Phytol. 150, 525-532.

Mauch-Mani, B. and Slusarenko, A.J. (1994) Systemic acquired resistance in Arabidopsis thaliana induced by a predisposing infection with a pathogenic isolate of Fusarium oxysporum. Mol. Plant Microbe Interact. 7, 378-383.

McGonigle, T.P., Miller, M.H., Evans, D.G., Fairchild, G.L. and Swan, J.A. (1990) A new method which gives an objective measure of colonization of roots by vesicular arbuscular mycorrhizal fungi. New Phytol. 115, 495-501.

Mellersh, D. and Parniske, M. (2006) Common symbiosis genes of Lotus japonicus are not required for intracellular accommodation of the rust fungus Uromyces loti. New Phytol. 170, 641-644.

Mitra, R.M., Gleason, C.A., Edwards, A., Hadfield, J., Downie, J.A., Oldroyd, G.E.D. and Long, S.R. (2004) A Ca2+/calmodulindependent protein kinase required for symbiotic nodule development: gene identification by transcript-based cloning. Proc. Natl Acad. Sci. USA, 101, 4701-4705.

Murray, M.G. and Thompson, W.F. (1980) Rapid isolation of high molecular weight plant DNA. Nucleic Acids Res. 8, 4321-4325.

Novero, M., Faccio, A., Genre, A., Stougaard, J., Webb, K.J., Mulder, L., Parniske, M. and Bonfante, P. (2002) Dual requirement of the LjSym4 gene for mycorrhizal development in epidermal and cortical cells of Lotus japonicus roots. New Phytol. 154, 741-749.

Ohtomo, R. and Saito, M. (2005) Polyphosphate dynamics in mycorrhizal roots during colonization of an arbuscular mycorrhizal fungus. New Phytol. 167, 571-578.

Oldroyd, G.E.D. and Downie, J.A. (2006) Nuclear calcium changes at the core of symbiosis signalling. Curr. Opin. Plant Biol. 9, 351357.

Parniske, M. (2004) Molecular genetics of the arbuscular mycorrhizal symbiosis. Curr. Opin. Plant Biol. 7, 414-421.

Paszkowski, U., Jakovleva, L. and Boller, T. (2006) Maize mutants affected at distinct stages of the arbuscular mycorrhizal symbiosis. Plant J. 47, 165-173.

Peterson, R.L. and Guinel, F.C. (2000) The use of plant mutants to study regulation of colonization by AM fungi. In Arbuscular
Mycorrhizas: Physiology and Function (Kapulnik, Y. and Douds, D.D., eds). Dordrecht: Kluwer Academic Publishers, pp. 147171.

Reinhardt, D. (2007) Programming good relations - development of the arbuscular mycorrhizal symbiosis. Curr. Opin. Plant Biol. 10, 98-105.

Saito, M. (2000) Symbiotic exchange of nutrients in arbuscular mycorrhizas: Transport and transfer of phosphorus. In Arbuscular Mycorrhizas: Physiology and Function (Kapulnik, Y. and Douds, D.D., eds). Dordrecht: Kluwer Academic Publishers, pp. 85-106.

Smith, S.E. and Read, D.J. (1997) Mycorrhizal Symbiosis, 2nd edn. London: Academic Press.

Stacey, G., Libault, M., Brechenmacher, L., Wan, J. and May, G.D. (2006) Genetics and functional genomics of legume nodulation. Curr. Opin. Plant Biol. 9, 110-121.

Stieger, P.A. and Feller, U. (1994) Nutrient accumulation and translocation in maturing wheat plants grown on waterlogged soil. Plant Soil, 160, 87-95.

Stracke, S., Kistner, C., Yoshida, S. et al. (2002) A plant receptor-like kinase required for both bacterial and fungal symbiosis. Nature, 417, 959-962.

Stuurman, J. and Kuhlemeier, C. (2005) Stable two-element control of dTph1 transposition in mutator strains of Petunia by an inactive ACT1 introgression from a wild species. Plant J. 41, 945-955.

Tisserant, B., Gianinazzi-Pearson, V., Gianinazzi, S. and Gollotte, A. (1993) In planta histochemical staining of fungal alkaline phosphatase activity for analysis of efficient arbuscular mycorrhizal infections. Mycol. Res. 97, 245-250.

Trouvelot, A., Kough, J.L. and Gianinazzi-Pearson, V. (1986) Mesure du taux de mycorhization VA d'un système radiculaire. Recherche de méthodes d'éstimation ayant une signification fonctionelle. In Les Mycorrhizes: Physiologie et Génétique. ESM/SEM, Dijon, 1-5 July (Gianinazzi, S. ed.), Paris: INRA Press, pp. 217-222.

Verwoerd, T.C., Dekker, B.M.M. and Hoekema, A. (1989) A smallscale procedure for the rapid isolation of plant RNAs. Nucleic Acids Res. 17, 2362-2362.

Weischenfeldt, J., Lykke-Andersen, J. and Porse, B. (2005) Messenger RNA surveillance: neutralizing natural nonsense. Curr. Biol. 15, R559-R562.

Wyss, P., Boller, T. and Wiemken, A. (1991) Phytoalexin response is elicited by a pathogen (Rhizoctonia solani) but not by a mycorrhizal fungus (Glomus mosseae) in soybean roots. Experientia, 47, 395-399.

Zhu, H., Riely, B.K., Burns, N.J. and Ané, J.-M. (2006) Tracing nonlegume orthologs of legume genes required for nodulation and arbuscular mycorrhizal symbioses. Genetics, 172, 2491-2499. 
TPJ-00322-2007

\section{Supplementary Table S1}

\begin{tabular}{|c|c|c|c|c|}
\hline F3 family & wt & mut & $\chi^{\mathbf{2}}$ & P \\
\hline No. 16 & 28 & 9 & 0.009 & $>0.92$ \\
\hline No. 17 & 27 & 11 & 0.32 & $>0.57$ \\
\hline No. 18 & 29 & 9 & 0.035 & $>0.85$ \\
\hline No. 21 & 31 & 9 & 0.133 & $>0.71$ \\
\hline No. 24 & 27 & 7 & 0.353 & $>0.55$ \\
\hline No. 25 & 25 & 12 & 1.09 & $>0.29$ \\
\hline No. 27 & 25 & 9 & 0.039 & $>0.84$ \\
\hline No. 106 & 27 & 10 & 0.081 & $>0.77$ \\
\hline No. 108 & 30 & 8 & 0.316 & $>0.57$ \\
\hline No. 110 & 29 & 8 & 0.225 & $>0.63$ \\
\hline No. 116 & 28 & 8 & 0.148 & $>0.70$ \\
\hline Total & $\mathbf{3 0 6}$ & $\mathbf{1 0 0}$ & & \\
\hline
\end{tabular}

\section{Legend}

\section{Supplementary Table S1}

Segregation ratios of progeny from stabilized heterozygous F2 plants from a cross of pam1 with the stabilizer line W5. Observed segregation ratios are consistent with a 3:1 segregation, suggesting that the pam 1 phenotype results from a recessive mutation in a single gene.

$\chi^{2}$ and $\mathrm{P}$ values are indicated for the individual families. 


\section{Supplementary material}

\section{Supplementary Table S1}

Segregation ratios of progeny from stabilized heterozygous F2 plants from a cross of pam1 with the stabilizer line W5.

$\chi^{2}$ and $\mathrm{P}$ values are indicated for the individual families.

\begin{tabular}{|c|c|c|c|c|c|c|c|}
\hline 3 weeks & Extrarad. & Intrarad. & Arbuscules & Appress. & aborted & Vesicles & Spores \\
\hline wt & $32 \pm 8.5$ & $12.5 \pm 2.1$ & $8 \pm 4.2$ & $0.5 \pm 0.7$ & 0 & $4 \pm 2.8$ & $0.5 \pm 0.7$ \\
\hline pam1 & $31.5 \pm 3.5$ & $15 \pm 4.2$ & $3 \pm 4.2$ & $7 \pm 8.5$ & $3 \pm 4.2$ & $3.5 \pm 4.9$ & 0 \\
\hline pam1S1 & $15 \pm 5.6$ & $9 \pm 2.8$ & 0 & $8.5 \pm 2.1$ & $7 \pm 2.8$ & 0 & 0 \\
\hline pam1S2 & $9.5 \pm 0.7$ & $2.5 \pm 2.1$ & 0 & $2.5 \pm 2.1$ & $2.5 \pm 2.1$ & 0 & 0 \\
\hline 5 weeks & Extrarad. & Intrarad. & Arbuscules & Appress. & aborted & Vesicles & Spores \\
\hline $\mathrm{wt}$ & $74 \pm 1.4$ & $77.5 \pm 3.5$ & $71.5 \pm 0.7$ & $28.5 \pm 2.1$ & 0 & $33.5 \pm 12.0$ & 0 \\
\hline pam1 & $40.5 \pm 48.8$ & $31.5 \pm 44.6$ & $24 \pm 33.9$ & $10 \pm 12.7$ & $2 \pm 1.41$ & $19.5 \pm 27.6$ & 0 \\
\hline pam1S1 & $10 \pm 7.1$ & $3 \pm 2.8$ & 0 & $4 \pm 2.8$ & $4 \pm 2.8$ & 0 & 0 \\
\hline pam1S2 & $6.5 \pm 6.4$ & $3.5 \pm 4.9$ & 0 & $2 \pm 1.4$ & $2 \pm 1.4$ & $0.5 \pm 0.7$ & 0 \\
\hline 8 weeks & Extrarad. & Intrarad. & Arbuscules & Appress. & aborted & Vesicles & Spores \\
\hline $\mathrm{wt}$ & $74 \pm 11.3$ & $77.5 \pm 9.2$ & $66.5 \pm 13.4$ & $21.5 \pm 0.7$ & 0 & $29 \pm 4.2$ & $1 \pm 1.4$ \\
\hline pam1 & $6.5 \pm 6.4$ & $5 \pm 5.6$ & 0 & $3.5 \pm 3.5$ & $3 \pm 2.8$ & $1 \pm 1.4$ & 0 \\
\hline pam1S1 & $3 \pm 0$ & 0 & 0 & $2 \pm 0$ & $2 \pm 0$ & 0 & 0 \\
\hline pam1S2 & $1.5 \pm 0.7$ & $2 \pm 0$ & 0 & 0 & 0 & 0 & 0 \\
\hline
\end{tabular}

Table S1: The dynamics of fungal colonization in pam1

Percent colonization is given for 3 successive time points in wild-type, pam1, and two stabilized mutant lines (pam1S1 and pam1S2). Total extraradical (Extrarad.) and intraradical (Intrarad.) colonization, as well as the percentage of the root system with arbuscules, appressoria (Appress.), aborted entries, vesicles and spores are given. 


\begin{tabular}{|l|c|c|c|c|c|c|}
\hline & Extrarad. & Intrarad.. & Arbuscules & Appress. & aborted & Vesicles \\
\hline wt soil & $25.7 \pm 6.11$ & $15.3 \pm 2.08$ & $12.3 \pm 2.08$ & $4.67 \pm 1.15$ & $0 \pm 0$ & $8.33 \pm 2.31$ \\
\hline Pam1S1 soil & $5.33 \pm 1.53$ & $0.67 \pm 1.15$ & $0 \pm 0$ & $0.33 \pm 0.58$ & $1.33 \pm 0.58$ & $0 \pm 0$ \\
\hline wt nurse & $66.3 \pm 8.02$ & $72.3 \pm 8.02$ & $44.3 \pm 2.52$ & $24.3 \pm 3.51$ & $0 \pm 0$ & $5.00 \pm 1.00$ \\
\hline Pam1S1 nurse & $48.3 \pm 4.73$ & $42.0 \pm 10.6$ & $0 \pm 0$ & $23.0 \pm 7.21$ & $7.67 \pm 2.52$ & $1.33 \pm 0.58$ \\
\hline
\end{tabular}

Table S2: Influence of inoculum identity on the dynamics of colonization in pam1S1

Percent colonization in wild-type and pam1S1 plants three weeks after inoculation with $G$. intraradices from whole-soil inoculum (soil) or from colonized wild-type plants (nurse) in separated compartments.

\begin{tabular}{|c|c|c|}
\hline & wt & pam1S1 \\
\hline PhPOLLUX & $100+/-16.4$ & $75.5+/-8.93$ \\
\hline PhSYMRK & $100+/-22.0 *$ & $71.8+/-7.84 *$ \\
\hline PhDMI3 & $100+/-15.3$ & $86.4+/-11.8$ \\
\hline
\end{tabular}

* Expression of PhSYMRK is significantly lower in pam1S1 compared to controls

Table S3: Relative expression of petunia SYM genes in wild-type and pam1S1 roots

Expression levels of PhPOLLUX, PhSYMRK, and PhDMI3 are expressed relative to GAPDH expression. Wild-type gene expression was normalized to 100 . 